\title{
BUDAN'S THEOREM FOR A CLASS OF ENTIRE FUNCTIONS
}

HERBERT S. WILF

I. Functions with negative real zeros. A classical theorem of Budan asserts that the number of zeros of the polynomial

$$
f(z)=a_{0}+a_{1} z+\cdots+a_{n} z^{n}
$$

in the interval $(a, b)$ is either equal to $V(a)-V(b)$, or less by an even number, where $V(x)$ is the number of variations of sign in the sequence

$$
f(x), f^{\prime}(x), \cdots, f^{(n)}(x) .
$$

It is easy to see that the "even number" qualification can be omitted if all the zeros of $f(z)$ are real, so that in this case we get exact information on every interval.

In this note we describe a class of entire functions for which the natural generalization of Budan's rule likewise gives exact information about the number of zeros in a real interval. This class $K$ is the set of entire functions $f(z)$ which are real for real $z$, have negative real zeros and positive Taylor coefficients, and are of finite exponential type. Such functions can be written in any of the forms

$$
\begin{array}{ll}
f(z)=\sum_{\nu=0}^{\infty} a_{\nu} z^{\nu}=\sum_{\nu=0}^{\infty} \frac{b_{\nu}}{\nu !} z^{\nu} & \left(a_{v}, b_{\nu}>0, \nu=0,1, \cdots\right), \\
f(z)=A e^{\alpha z} \prod_{n=1}^{\infty}\left(1+\frac{z}{z_{n}}\right) e^{-z / z_{n}} & \left(z_{n}>0, n=1,2, \cdots\right), \\
f(z)=A \prod_{n=1}^{\infty}\left(1+\frac{z}{z_{n}}\right) & \left(z_{n}>0, n=1,2, \cdots\right) .
\end{array}
$$

Lemma 1. Let $f(z) \in K$. Then

$$
z_{1}^{2} \geqq a_{0}^{2}\left(a_{1}^{2}-2 a_{0} a_{2}\right)^{-1} .
$$

Proof. Suppose $f(z)$ has the form (II)', i.e., is of genus one. Then

$$
\frac{f^{\prime}(z)}{f(z)}=\alpha-\sum_{n=1}^{\infty} \frac{z}{z_{n}} \frac{1}{1+z / z_{n}}=\alpha+\sum_{m=1}^{\infty}(-1)^{m} \sigma_{m+1} z^{m}
$$

where

$$
\sigma_{m}=\sum_{n=1}^{\infty} z_{n}^{-m} \quad(m \geqq 2)
$$

Received by the editors February 2, 1961. 
and the representation (2) converges for $|z|<z_{1}$. Matching coefficients in (2)

$$
\sigma_{2}=a_{0}^{-2}\left(a_{1}^{2}-2 a_{0} a_{2}\right)
$$

and since $\sigma_{2} \geqq z_{1}^{-2}$, the result follows. ${ }^{1}$ If $f(z)$ is of genus zero the proof is identical.

Lemma 2. Let $f(z) \in K$, and let $-z_{p 1}$ denote the zero of $f^{(p)}(z)$ nearest the origin. Then, as $p \rightarrow \infty, z_{p 1} \rightarrow \infty$.

Proof. Applying Lemma 1 to $f^{(p)}(z) \in K$, we find

$$
\begin{aligned}
z_{p 1}^{2} & \geqq \frac{a_{p}^{2}}{p+1}\left\{(p+1) a_{p+1}^{2}-(p+2) a_{p} a_{p+2}\right\}^{-1} \\
& =\frac{b_{p}}{b_{p+1}}\left\{\frac{b_{p+1}}{b_{p}}-\frac{b_{p+2}}{b_{p+1}}\right\}^{-1} \quad(p=0,1,2, \cdots) .
\end{aligned}
$$

The quantity in braces is well known to be positive [2, p. 24] and therefore the bound is nontrivial. Further, since

$$
\frac{b_{p+2}}{b_{p+1}}<\frac{b_{p+1}}{b_{p}}
$$

the sequence $\left\{b_{p+1} / b_{p}\right\}_{0}^{\infty}$ is a decreasing sequence of positive numbers, and therefore approaches a limit, which by the ratio test is actually the type $\tau$ of $f(z)$. It is now evident that the right member of (3) tends to $+\infty$ as $p \rightarrow \infty$, proving the lemma.

Next, if $f(z) \in K$ it is well known that $[2$, p. 24]

$$
f^{(p-1)}(x) f^{(p+1)}(x)<\left\{f^{(p)}(x)\right\}^{2}
$$

and hence that at any zero of $f^{(p)}(x), f^{(p-1)}(x)$ and $f^{(p+1)}(x)$ have opposite signs. Let $(a, b)$ be a fixed, finite interval of the real axis. By Lemma 2 , there is an integer $p_{0}$ such that for $p \geqq p_{0}$, the interval $(a, b)$ is free of zeros of $f^{(p)}(z)$, i.e., $f^{(p)}(z)$ is of constant sign on $(a, b)$. For $p \geqq p_{0}$ then, the sequence (e.g. [5]) $f(x), f^{\prime}(x), \cdots, f^{(p)}(x)$ is a Sturm sequence for $(a, b)$. Hence if $V\left(\xi_{1}, \xi_{2}, \cdots, \xi_{n}\right)$ denotes the number of changes of sign in the sequence $\xi_{1}, \xi_{2}, \ldots, \xi_{n}$, the number of zeros of $f(z)$ in $(a, b)$ is

$V\left(f(a), f^{\prime}(a), \cdots, f^{(p)}(a)\right)-V\left(f(b), f^{\prime}(b), \cdots, f^{(p)}(b)\right) \equiv V_{p}(a)-V_{p}(b)$

provided $p \geqq p_{0}$. We have shown

THEOREM 1. Let $f(z) \in K$. Then the number of zeros of $f(z)$ in $(a, b)$ is precisely

${ }^{1}$ The idea goes back to Euler; compare [1, p. 500]. 


$$
\lim _{n \rightarrow \infty}\left\{V_{n}(a)-V_{n}(b)\right\} \text {. }
$$

We note that for $n$ large enough, $V_{n}(a)-V_{n}(b)$ is constant, and that, in particular cases, the size of $n$ which is "large enough" can be estimated from (3).

Taking $b=0$, we find easily

TheOREM 2. Let $f(z) \in K$ have the Taylor expansion

$$
f(z)=\sum_{\nu=0}^{\infty} c_{\nu}(z+a)^{\nu}
$$

about $z=-a$. Then the number of zeros of $f(z)$ in $(-a, 0)$ is exactly the number of changes of sign in the coefficient sequence $\left\{c_{\nu}\right\}_{0}^{\infty}$.

II. Generalizations. The hypotheses of positive coefficients and negative real zeros were really necessary only to insure that $z_{1 p} \rightarrow \infty$. Hence the conclusion of Theorem 1 remains true for functions of order $\rho<2$ with only real zeros (of arbitrary signs) and for which $\left|z_{1 p}\right| \rightarrow \infty$. The behavior of $\left|z_{1 p}\right|$ has been well studied (e.g. $\left.[3 ; 4]\right)$ with the conclusion that $\left|z_{1 p}\right|$ does not $\rightarrow \infty$ if $\rho>1$, and need not if $\rho \leqq 1$, as the example $\sin \tau z$ shows.

Gontcharoff [4] has shown, however, that if $\rho<1$, or if $\rho=1, \tau=0$, then, for any fixed interval $(a, b)$ there is a sequence $\left\{p_{k}\right\}$ tending to infinity such that $f^{\left(p_{k}\right)}(z)$ is not zero in $(a, b)$. Hence the number of zeros of a function of zero exponential type (including $\rho<1$ ) with only real zeros is exactly

$$
V_{p_{k}}(a)-V_{p_{k}}(b),
$$

$k$ being arbitrary. In the absence of any knowledge of the sequence $\left\{p_{k}\right\}$, all we can say is that among the numbers $\left\{V_{n}(a)-V_{n}(b)\right\}_{0}^{\infty}$, the number of zeros of $f(z)$ in $(a, b)$ appears infinitely often, if $f(z)$ is of zero exponential type with only real zeros.

In conclusion we give another example of a situation where the zeros of $f^{(k)}(z)$ ultimately lie outside of any fixed compact set, namely the case where $f(z)$ is the product of $e^{-\alpha z}$ by a polynomial. The main interest of this result lies in the fact that the asymptotic distribution of all the zeros of the $k$ th derivative is independent of the polynomial chosen.

THEOREM 3. Let

$$
P(z)=\sum_{\nu=0}^{p} a_{\nu} z^{\nu}=\sum_{\nu=0}^{p} \frac{b_{\nu}}{\nu !} z^{\nu}
$$

be a given polynomial, and let $P_{n}(z)$ be defined by 


$$
\left(\frac{d}{d z}\right)^{n}\left\{e^{-z} P(z)\right\}=(-1)^{n} e^{-z} P_{n}(z)
$$

Then

$$
\frac{1}{a_{p}} \lim _{n \rightarrow \infty}\left\{n^{-p} P_{n}(n z)\right\}=(z-1)^{p} .
$$

Proof. We have

$$
\left(\frac{d}{d z}\right)^{n}\left\{e^{-z} P(z)\right\}=e^{-z} \sum_{k=0}^{n} C_{n, k}(-1)^{n-k} P^{(k)}(z)
$$

and therefore for $n \geqq p$,

$$
\begin{aligned}
P_{n}(z) & =\sum_{k=0}^{p} C_{n, k}(-1)^{k} P^{(k)}(z) \\
& =\sum_{r=0}^{p} \frac{z^{r}}{r !} \sum_{k=0}^{p-r}(-1)^{k} C_{n, k} b_{r+k} \equiv \sum_{r=0}^{p} \frac{b_{r}^{(n)}}{r !} z^{r} .
\end{aligned}
$$

Hence for each fixed $\nu$,

$$
b_{p \rightarrow \nu}^{(n)} \sim \frac{n^{\nu}}{\nu !}(-1)^{\nu} b_{p} \quad \cdot(n \rightarrow \infty)
$$

and the result follows by an obvious calculation.

In particular, if $z_{1 k}, z_{2 k}, \cdots, z_{p k}$ are the zeros of the $k$ th derivative of $e^{-z} P(z)$, where $P(z)$ is any polynomial of degree $p$, then for each fixed $\nu, z_{v k} \sim k(k \rightarrow \infty)$. As an example, if $p=2$, a more exact calculation shows that the zeros of $P_{n}(z)$ are

$$
n \pm n^{1 / 2}+O(1) \quad(n \rightarrow \infty)
$$

the dependence on $P(z)$ being $O(1)$.

\section{REFERENCES}

1. G. N. Watson, A treatise on the theory of Bessel functions, Cambridge University Press, 1958.

2. R. P. Boas, Jr., Entire functions, Academic Press, New York, 1954.

3. G. Polya, On the zeros of the derivatives of a function and its analytic character, Bull. Amer. Math. Soc. 49 (1943), 178-191.

4. M. W. Gontcharoff, Recherches sur les derivees successives des fonctions analytiques, Ann. Sci. de L'École Norm. Sup. 47 (1930), 1-92.

5. L. E. Dickson, $A$ first course in the theory of equations, Wiley, New York, 1922.

The University OF IlLiNois 